\title{
Bias reduction in traceroute sampling: towards a more accurate map of the Internet
}

\author{
Abraham D. Flaxman ${ }^{1}$ and Juan Vera ${ }^{2}$ \\ 1 Microsoft Research \\ Redmond, WA \\ abie@microsoft.com \\ ${ }^{2}$ Georgia Institute of Technology \\ Atlanta, GA \\ jvera@cc.gatech.edu
}

\begin{abstract}
Traceroute sampling is an important technique in exploring the internet router graph and the autonomous system graph. Although it is one of the primary techniques used in calculating statistics about the internet, it can introduce bias that corrupts these estimates. This paper reports on a theoretical and experimental investigation of a new technique to reduce the bias of traceroute sampling when estimating the degree distribution. We develop a new estimator for the degree of a node in a traceroute-sampled graph; validate the estimator theoretically in Erdős-Rényi graphs and, through computer experiments, for a wider range of graphs; and apply it to produce a new picture of the degree distribution of the autonomous system graph.
\end{abstract}

\section{Introduction}

The internet is quite a mysterious network. It is a huge and complex tangle of routers, wired together by millions of edges. To understand this router graph is quite a challenge, one that has driven research for the last decade.

The router graph has a natural clustering into Autonomous Systems (ASes), which are sets of routers under the same management. Producing an accurate picture of the $A S$ graph is an important step towards understanding the internet.

There are three techniques for finding large sets of edges in the AS graph: the WHOIS database, BGP tables, and traceroute sampling. No approach is clearly superior, and the results of the different approaches are compared in detail in a recent paper [14].

The present paper focuses on traceroute sampling, an approach applicable to the router graph as well as the AS graph. Traceroute sampling consists of recording the paths that packets follow when they are sent from monitor nodes to target nodes, and merging all of these paths to produce an approximation of the AS graph.

A seminal analysis using both traceroute sampling and BGP tables concluded that the AS graph degree distribution follows a power-law (meaning that the number of ASes of degree $k$ is proportional to $k^{-\alpha}$ for a wide range of $k$ values) [7]. This caused a shift in simulation methodology for evaluating network algorithms and also contributed to the avalanche of recently developed network models which produce power-law degree distributions.

However, the true nature of the AS-graph degree distribution was called into question by computer experiments on synthetic graphs [12,17]. These experiments show that if the sets of monitor and target nodes are too small then traceroute sampling will produce a power-law degree distribution, even when the underlying graph has a tightly concentrated degree distribution. Theoretical follow-up work proved rigorously that in many non-power-law graphs, including random regular graphs, an idealized model of traceroute sampling yields power-law degree distributions $[4,1]$.

Subsequent computer experiments have led some to believe that the bias inherent to traceroute sampling can be ignored, at least for making a qualitative distinction between scale-free and homogeneous graphs, when using a large enough set of monitor nodes [9]. This is also supported by an analysis using the statistical physics technique of mean field approximation [5]. 


\subsection{Our contribution}

This paper proposes a new way forward in the struggle to characterize the degree distribution of the AS graph. Our contribution has three parts:

1. We derive a statistical technique for reducing the bias in traceroute sampling;

2. We verify the technique experimentally and theoretically, in the framework previously studied in $[12,4]$;

3. We use the traceroute bias-reduction technique to generate a more accurate picture of the AS degree distribution over time, which suggests that aspects of commercially available technology are reflected in the network topology.

Our approach for reducing the bias in traceroute sampling is based on a technique from biostatistics, the multiple-recapture census, which has been developed for estimating the size of an animal population [18] (this technique also has applications to proofreading [19]). However, we do not have the benefit of independent random variables which are central to the animal counting and proofreading statistics, and so we must adapt the technique to apply to random variables with complicated dependencies.

To provide some evidence that this bias-reduction technique actually reduces bias, we consider a widely used model of traceroute sampling, which assumes that data travels from monitor to target along the shortest path in the network. It is generally believed that the path that data actually takes is not the shortest path, but that the shortest path is an acceptable approximation of the actual path (see [13] for a discussion of this approximation). In this model, it is possible to check theoretically and experimentally that the bias reduction provides a better estimate of the degree distribution. We show that the new estimation is asymptotically unbiased for the Erdős-Rényi random graph $G_{n, p}$ when $n p \gg \log n$, and that it gives improved estimates for finite instances from a variety of different graphs.

Finally, we use the bias-reduction technique on real data, traceroute samples from the internet. The new estimate of the AS-graph degree distribution is still scale-free over two orders of magnitude, with an exponent very similar to the uncorrected degree distribution (see Figure 1). A by-product of bias reduction is the removal of all vertices with degree less than 3, and this increases the average degree. For example, in March 2004 (the month used for comparison in [14]), the biased estimate of average degree is 6.29, while after bias reduction the average degree is 12.66 (which is very close to 12.52, the biased average degree when restricted to vertices of degree at least 3). An interesting feature in the bias-reduced AS degree distribution (from March 2004) is the lack of nodes with degree between 65 and 90; at the time, a popular router maker offered a router which provided up to 64 ports per chassis. In March 2002, before this product was available, there was no dearth of 65 degree nodes.

\subsection{Related work}

Internet mapping by traceroute sampling was pioneered by Pansiot and Grad in [15], and the scale-free nature of the degree distribution was observed by Faloutsos, Faloutsos, and Faloutsos in [7]. Since 1998, the Cooperative Association for Internet Data Analysis (CAIDA) project skitter has archived traceroute data that is collected daily [10]. The bias introduced by traceroute sampling was identified in computer experiments by Lakhina, Byers, Crovella, and Xie in [12] and Petermann and De Los Rios [17], and formally proven to hold in a model of one-monitor, all-target traceroute sample by Clauset and Moore [4] and, in further generality, by Achlioptas, Clauset, Kempe, and Moore [1]. Computer experiments by to Guillaume, Latapy, and Magoni [9] and an analysis using the mean field approximation of statistical physics due to Dall'Asta, Alvarez-Hamelin, Barrat, Vázquez, and Vespignani [5] argue that, despite the bias introduced by traceroute sampling, some sort of scale-free behavior can be inferred from the union of traceroute-sampled paths.

The present paper provides a new avenue for investigating these controversial questions, by developing a method for correcting the bias introduced by traceroute sampling. Another recent paper by Viger, Barrat, Dall'Asta, Zhang and Kolaczyk applied techniques from statistics to reduce the bias of traceroute sampling [21]. That paper focused on estimating the number of nodes in the AS graph, and applied techniques from a different problem in biostatistics, estimating the number of species in a bioregion. The problem of correcting 


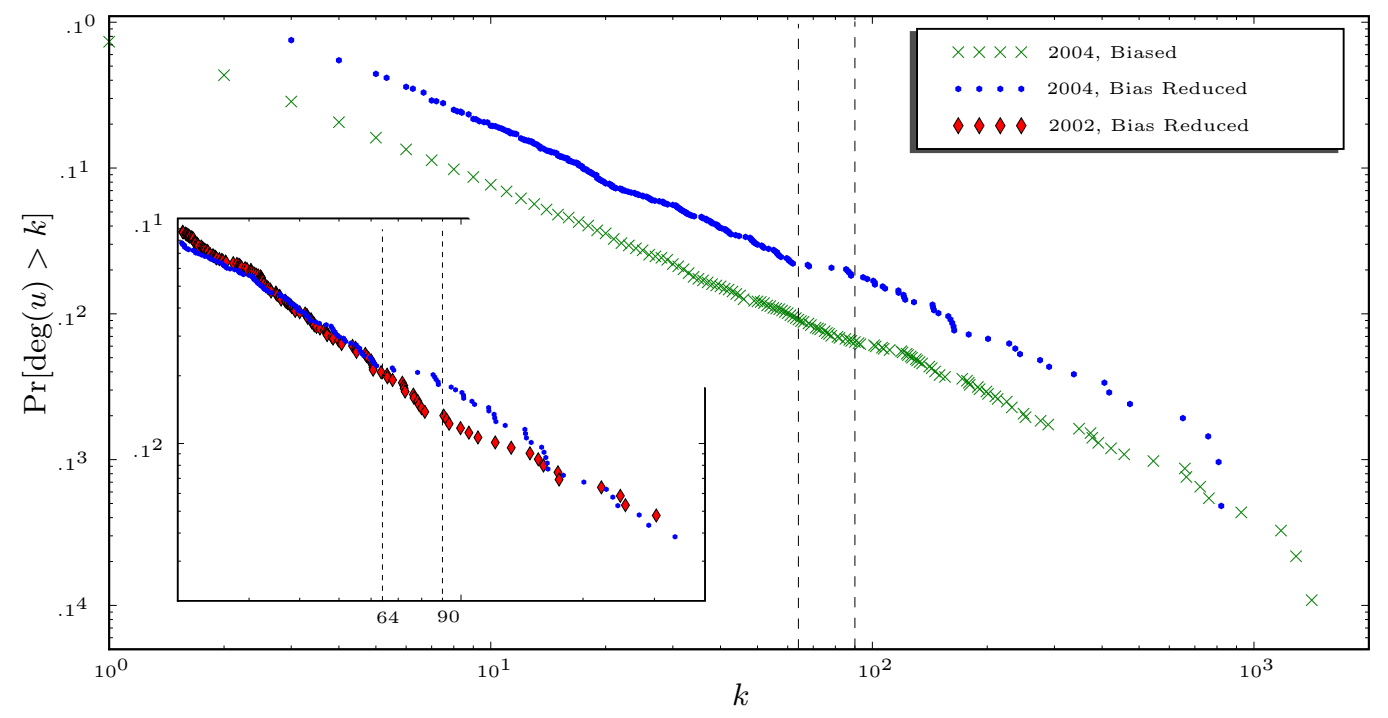

Fig. 1. Degree sequence ccdf estimates for the AS graph (from CAIDA skitter). Main panel: March, 2004, with and without bias reduction. Inset: a portion of ccdf for March, 2004 and March, 2002, both with bias reduction. The nodes with degree between 65 and 90 in 2002 have disappeared in 2004.

bias in sampled networks has a long history in sociology, although the biases in that domain seem somewhat different; see the surveys by Frank, by Klovdahl, or by Salganik and Heckathorn for an overview $[8,11,20]$.

In addition to traceroute sampling, maps of the AS graph have been generated in two different ways, using BGP tables and using the WHOIS database. A recent paper by Mahadevan, Krioukov, Fomenkov, Dimitropoulos, claffy, and Vahdat provides a detailed comparison of the graphs that result from each of these measurement techniques [14].

\subsection{Outline of what follows}

The new estimator for the degree of a node in the AS graph is developed from multiple-recapture population estimation in Section 2. Section 3 argues that this estimator generates an asymptotically unbiased degree distribution for the Erdős-Rényi graph $G_{n, p}$ when $p \gg \log n$, which rigorously demonstrates that the new estimator can reject a null hypothesis. Section 4 presents additional evidence that the new estimator reduces the bias of traceroute sampling, in the form of computer experiments on synthetic networks. Section 5 provides a comparison between the degree sequence predicted by the new estimator and the previous technique, and details how, after bias reduction, the degree distribution may reflect economic and technological factors present in the system, i.e., there a significantly larger marginal cost of adding a 65 th neighbor than adding a 64th neighbor when using the Juniper T320 edge router. Section 6 provides a conclusion and focuses on directions of future research to strengthen this approach.

\section{Estimation Technique}

The classical capture-recapture approach to estimating an animal population has two phases. First, an experimenter captures animals for a given time period, marks them (with tags or bands), and releases them, recording the total number of animals captured. Then, the experimenter captures animals for a second time 
period, and records both the number of animals recaptured and the total number of animals captured during the second period. If $A$ denotes the number of animals captured in phase one, $B$ denotes the number captured during phase two, and $C$ denotes the number captured in phase one and captured again in phase two, then an estimate of total population size is given by

$$
\widehat{N}= \begin{cases}\frac{A B}{C}, & \text { if } C \neq 0 \\ \infty, & \text { otherwise }\end{cases}
$$

If the true population size is $N$, and each animal is captured or not captured during each phase independently, with probability $p_{1}$ during phase one and probability $p_{2}$ during phase two, then $\widehat{N}$ is the maximum likelihood estimate of $N$ [18]. For more than two phases, the maximum likelihood estimator does not have a simple closed form, but it can be computed efficiently using the techniques developed in [18].

When estimating the degree of a particular AS by traceroute sampling, each edge corresponds to an animal, and each monitor node corresponds to a recapture phase. Unfortunately, in this setting there is no reason to believe that the events "monitor $i$ observes edge $j$ " are independent. Indeed, when shortest-path routing is used (as an approximation of BGP routing), these events are highly dependent. However, it is still possible adapt the capture-recapture estimate to reduce bias in this case.

Let $G$ be a graph, and let $s$ and $t$ be monitor nodes in $G$. Let $G_{s}$ be the union of all routes discovered when sending packets from $s$ to every node in the target set. Define $G_{t}$ analogously. Let $N_{s}(u)$ denote the neighbors of $u$ in $G_{s}$ and define $N_{t}(u)$ analogously.

Using this notation, the modification of the capture-recapture estimate proposed for traceroute sampling is given by

$$
\widehat{\operatorname{deg}}_{s, t}(u)= \begin{cases}\frac{\left|N_{s}(u)\right| \cdot\left|N_{t}(u)\right|}{\left|N_{s}(u) \cap N_{t}(u)\right|}, & \text { if }\left|N_{s}(u) \cap N_{t}(u)\right|>2 ; \\ \infty, & \text { otherwise. }\end{cases}
$$

When more than 2 monitor nodes are available, pair up the monitors, consider the estimates given by each pair that are not $\infty$, and for the final estimator, use the median of these values. So, if the monitor nodes are paired up as $\left(s_{1}, t_{1}\right),\left(s_{2}, t_{2}\right), \ldots,\left(s_{k}, t_{k}\right)$ then

$$
\widehat{\operatorname{deg}}(u)=\operatorname{median}\left(\left\{\widehat{\operatorname{deg}}_{s_{i}, t_{i}}(u) \neq \infty\right\}\right) .
$$

This degree estimator can also provide an estimate of the cdf of the degree distribution (i.e., the fraction of nodes with degree at most $k$ ) according to the formula

$$
\widehat{d_{\leq k}}=\widehat{\operatorname{Pr}}[\operatorname{deg}(u) \leq k]=\frac{\#\left\{u: \widehat{\operatorname{deg}}_{s, t}(u) \leq k\right\}}{\#\left\{u: \widehat{\operatorname{deg}}_{s, t}(u)<\infty\right\}}
$$

Discussion: It may seem wasteful to consider the median-of-two-monitors estimate instead of combining all available monitors in a more holistic manner. However, we have conducted computer experiments with maximum likelihood estimators for multiple-recapture population estimation with more than two phases, and the adaptations we have considered thus far perform significantly worse than the median-of-two-monitors approach above. This is probably due to the complicated dependencies of several overlapping shortest-path trees. However, the exploration we have conducted to date is not exhaustive, and does not rule out the possibility that a significantly better estimator exists.

\section{Theoretical analysis}

This section and the next intend to provide some assurance that repeated application of $\widehat{\operatorname{deg}}(u)$ is an accurate way to estimate the degree distribution of the sampled graph.

This section provides a theoretical analysis of the performance of $\widehat{\operatorname{deg}}(u)$ in a very specific setting: when the underlying graph is the Erdös-Rényi graph $G_{n, p}$ with $n$ sufficiently large, $n p \gg \log n$, and every vertex is a target node. For the purpose of analysis, this section and the next assume that traceroute finds a shortest path from monitor to target. This is the same setting that is considered in [4]. 
Theorem 1. Let $G \sim G_{n, p}$ be a random graph with $n p=d \gg \log n$, and let $s, t$, and $u$ be uniformly random vertices of $G$. Then, for any $k$, with high probability,

$$
\widehat{d_{\leq k}}=\frac{\#\{u: \widehat{\operatorname{deg}}(u) \leq k\}}{\#\{u: \widehat{\operatorname{deg}}(u)<\infty\}}=\frac{\#\{u: \operatorname{deg}(u) \leq k\}}{n} \pm \mathcal{O}(1 / d) .
$$

Proof sketch: The analysis two breadth-first-search trees in a random graph is difficult when the average degree is small. But, for $d$ moderately large, as in this theorem, the situation is simpler.

It follows from the branching-process approximation of breadth-first search that with high probability there are $(1 \pm \epsilon) d^{i}$ vertices at distance exactly $i$ from $s($ or $t)$ when $i<(\log n) /(\log d)$. Thus, almost all vertices are distance $\lceil(\log n) /(\log d)\rceil$ apart. For ease of analysis, suppose that $\ell=(\log n) /(\log d)$ is an integer.

So, with high probability, if $u$ is at distance $\ell$ from $s$ or $t$ then it is a leaf node in $G_{s}$ or $G_{t}$. In this case, $\left|N_{s}(u) \cap N_{t}(u)\right| \leq 1$ and therefore $\widehat{\operatorname{deg}}(u)=\infty$.

Now, consider the case where vertex $u$ is distance $i$ from $s$ and distance $j$ from $t$, where $i, j<\ell$. Let $N(u)$ denote the neighbors of $u$ in $G$, and then let $S$ be the set of vertices within distance $i$ of $s$ in $G$ and let $T$ be the set of vertices within distance $j$ of $t$ in $G$. Conditioned on $S, T$ and $N(u)$, the set of indicator random variables

$$
\left\{\mathbf{1}\left[v \in N_{s}(u)\right], \mathbf{1}\left[v \in N_{t}(u)\right]: v \in N(u) \backslash(S \cup T)\right\}
$$

is independent, and, for $v \in N(u) \backslash(S \cup T), \operatorname{Pr}\left[v \in N_{s}(u)\right]$ and $\operatorname{Pr}\left[v \in N_{t}(u)\right]$ are functions of $S$ and $T$, but constants with respect to $v$, i.e., $\operatorname{Pr}\left[v \in N_{s}\right]=p_{s}$ and $\operatorname{Pr}\left[v \in N_{t}\right]=p_{t}$. So, besides any edges between $u$ and $S \cup T$, the edges incident to $u$ in $G_{s}[S]$ and $G_{t}[T]$ yield the random variables $\left|N_{s}(u)\right|,\left|N_{t}(u)\right|$, and $\left|N_{s}(u) \cap N_{t}(u)\right|$, which correspond to $A, B$, and $C$ in the capture-recapture estimate of population size. For example, if there is only one edge incident to $u$ in $G_{s}[S]$ and only one in $G_{t}[T]$, and these edges are different, then

$$
\operatorname{Pr}[\widehat{\operatorname{deg}(u)} \geq k \mid S, T, N(u)]=\operatorname{Pr}\left[\frac{(A+1)(B+1)}{C} \geq k\right],
$$

where $C \sim \mathrm{B}\left(|N(u)|-2, p_{s} p_{t}\right), A \sim C+\mathrm{B}\left(|N(u)|-1-C, p_{s}\right)$, and $B \sim C+\mathrm{B}\left(|N(u)|-1-C, p_{t}\right)$. If $k$ is sufficiently large and $p_{s}$ and $p_{t}$ are not too small then this probability is concentrated in the range $k=|N(u)| \pm \sqrt{|N(u)|}$.

To complete the proof, it remains to show that, with probability $1-\mathcal{O}(1 / d), p_{s}, p_{t} \geq \epsilon$ and $\mid N(u) \cap(S \cup$ $T) \mid \leq 2$, and from this show that, for $A, B, C$ defined analogously to above,

$$
\operatorname{Pr}\left[\frac{(A+1)(B+1)}{C} \geq k\right]=\operatorname{Pr}[|N(u)| \geq k]+\mathcal{O}(1 / d) .
$$

Discussion: This analysis would go through without modification if the estimate also included samples where $\left|N_{s}(u) \cap N_{t}(u)\right|=2$, but the definition of $\widehat{\operatorname{deg}}(u)$ from above seems to behave better under finite scaling.

The proof sketch can be adapted for random graphs with other degree distributions, provided that the average degree is large. However, the proof relies on the fact that the graph is locally tree-like, which ensures that $N(u) \cap(S \cup T)$ is likely to be small. This assumption does not seem to hold in the AS graph, and even $G_{s}$, the union of all routes discovered from a single monitor node $s$, has some triangles. The next section includes evidence from computer experiments that in graphs which are not locally tree-like, such as the random geometric graph, estimator $\widehat{\operatorname{deg}}(u)$ is not asymptotically unbiased, but can still reduce some amount of bias. Proving this rigorously may be a difficult task.

\section{Computer experiments}

This section describes the results of a series of computer experiments conducted to investigate how well $\widehat{d_{\leq k}}$ approximates the true degree distribution. 
We consider three different distributions for random graphs, the Erdös-Rényi model, the Preferential Attachment model, and the random geometric graph. Additionally, we consider synthetic data based on a real-world graph, the Western States Power Grid (WSPG), which Duncan Watts has graciously made available to researchers [22]. These graphs will all be described in more detail below.

For each graph, we set edge $e$ to be of length $1+\eta_{e}$, where $\eta_{e}$ is selected uniformly from the interval $[-1 / n, 1 / n]$, where $n$ is the number of vertices. This ensures that there are not multiple shortest paths between pairs of vertices. We approximate the path that data takes from a monitor to a target node by the shortest path. This follows the experimental design of [12].

For each graph distribution, and for a range of graph sizes, edge densities, monitor set sizes, and target set sizes, we estimate the degree of every vertex by $\widehat{\operatorname{deg}}(u)$ and by the biased estimator given by the union of the edges discovered by traceroute sampling,

$$
\widehat{\operatorname{deg}}_{\text {biased }}(u)=\left|\bigcup_{s \in V_{m}} N_{s}(u)\right|,
$$

where $V_{m}$ is the set of monitor nodes and $N_{s}(u)$ denotes the neighbors of $u$ in the union of all routes discovered when sending packets from $s$ to every node in the target set $V_{t}$. This provides estimates of the degree distribution cdf, by the reduced bias estimator $\widehat{d_{\leq k}}$ from above and by the biased estimator $\widehat{d}_{\leq k}$ biased , defined by

$$
\widehat{d \leq k}_{\text {biased }}=\frac{\#\left\{u: \widehat{\operatorname{deg}}_{\text {biased }}(u) \leq k\right\}}{\#\left\{u: \widehat{\operatorname{deg}}_{\text {biased }}(u) \geq 1\right\}} .
$$

$\widehat{d \leq k}_{\text {biased }}$ has been the primary approach considered in prior work.

We use these estimates to calculate the $\ell_{2}$ error of the degree distribution cdf estimate, given by

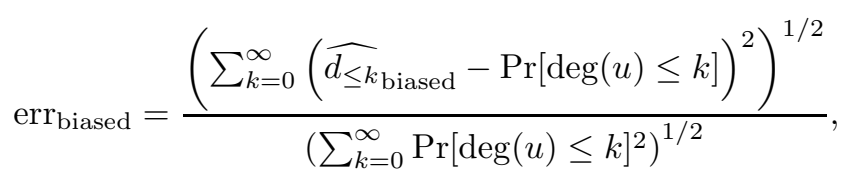

and

$$
\operatorname{err}_{\text {reduced }}=\frac{\left(\sum_{k=0}^{\infty}\left(\widehat{d_{\leq k}}-\operatorname{Pr}[\operatorname{deg}(u) \leq k]\right)^{2}\right)^{1 / 2}}{\left(\sum_{k=0}^{\infty} \operatorname{Pr}[\operatorname{deg}(u) \leq k]^{2}\right)^{1 / 2}},
$$

where $\operatorname{Pr}[\operatorname{deg}(u) \leq k]=\#\{u: \operatorname{deg}(u) \leq k\} / n$ is the probability with respect to a uniformly random choice of $u$ from the vertices of $G$.

We also exhibit plots of the distribution and the two estimates for a typical parameter setting. All error values reported are the median value of 100 experiments, and the plots show the distribution with the median error as well as the pointwise 90th percentile values from the 100 experiments.

\subsection{Random graph, $G_{n, m}$}

The Erdös-Rényi distribution of graphs, $G_{n, m}$, can be generated by choosing a graph uniformly at random from all graphs with $n$ vertices and $m$ edges [6]. It was not developed to model real-world graphs, but it is analytically tractable and can provide insight into the behavior of more realistic graph models. It can also be used as a null hypothesis. Section 3 proved that $\widehat{\operatorname{deg}}(u)$ and $\widehat{d_{\leq k}}$ are asymptotically unbiased for $G_{n, p}$ when $n p \gg \log n$. Conventional wisdom holds that anything true for $G_{n, p}$ is also true for $G_{n, m}$ when $m \approx\left(\begin{array}{l}n \\ 2\end{array}\right) p$, and computer experiments support this conclusion, even for moderately size $n$ and $m$, as shown in Table 1 and Figure $2 \mathrm{a}$. These experiments indicate that $\widehat{\operatorname{deg}}(u)$ and $\widehat{d_{\leq k}}$ are also good estimators when the number of targets $n_{t}$ is a reasonably small fraction of $n$, which is the case in traceroute sampling of the AS graph. 


\begin{tabular}{|r|r|r|r|r|r|}
\hline$n$ & $d$ & $n_{m}$ & $n_{t}$ & $\%$ err $_{\text {biased }}$ & \% err $_{\text {reduced }}$ \\
\hline 1,000 & 15 & 2 & $n / 8$ & 3.38 & 3.15 \\
& & & $n / 2$ & 3.08 & 0.96 \\
& & & $n$ & 2.81 & 0.42 \\
& & 8 & $n / 2$ & 2.11 & 0.81 \\
& & 16 & $n / 2$ & 1.38 & 0.80 \\
\hline 10,000 & 20 & 2 & $n / 8$ & 4.02 & 2.10 \\
& & & $n / 2$ & 3.75 & 1.25 \\
& & & $n$ & 3.51 & 0.46 \\
\hline 100,000 & 15 & 2 & $n$ & 2.81 & 0.21 \\
\hline
\end{tabular}

Table 1. $\ell_{2}$ error in degree distribution estimation with and without bias reduction for Erdős-Rényi graph, $G_{n, m}$ where $d=2 m / n$, with $n_{m}$ monitors and $n_{t}$ targets (median values of 100 trials).

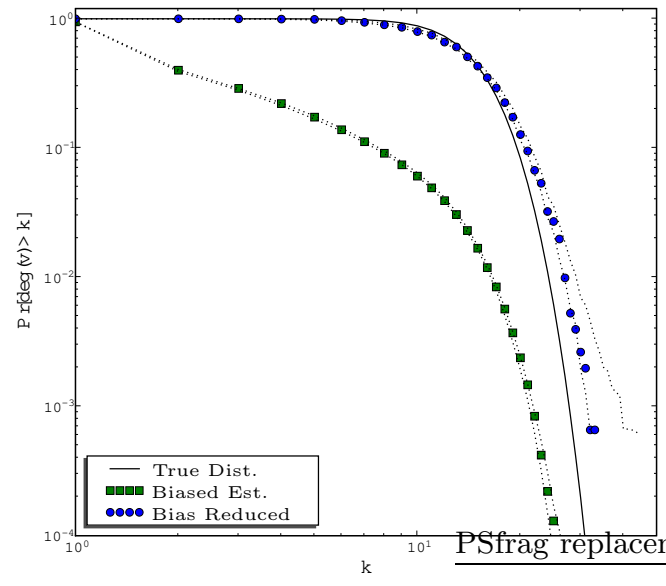

(a) $G_{n, m}$ with $n=100,000, d=2 m / n=15$.

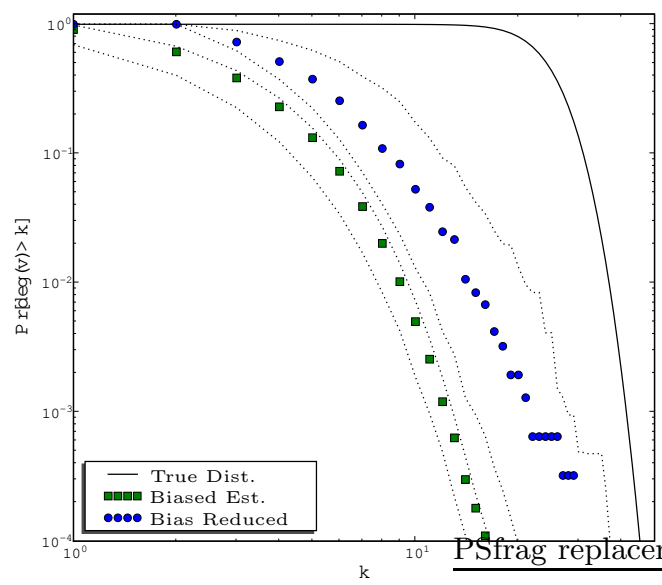

(c) $G(\mathcal{X} ; r)$ with $n=100,000, d=\pi r^{2}=25$.

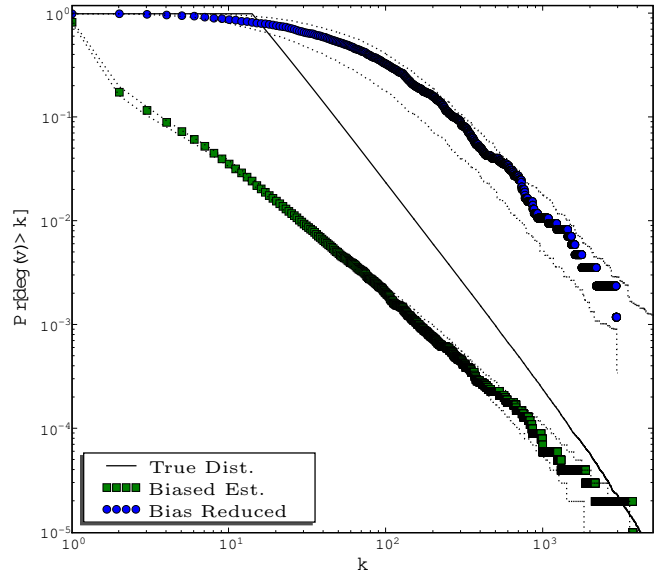

(b) PA graph with $n=100,000, m=15$.

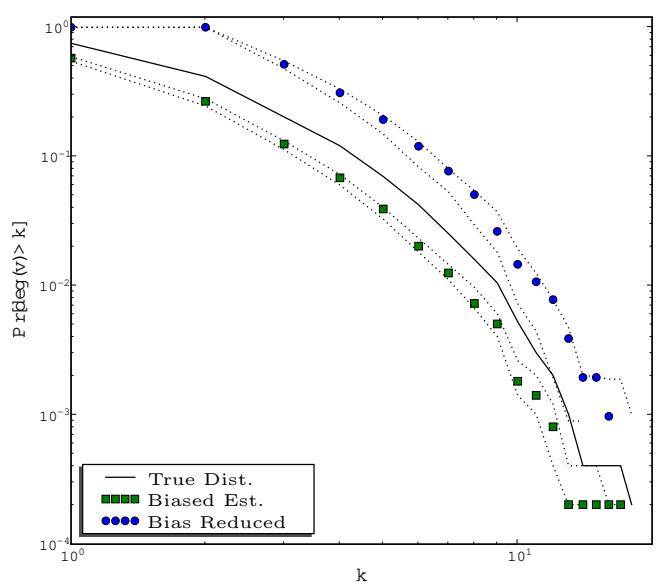

(d) Western states power graph from [22].

Fig. 2. Degree sequence ccdf, biased, and bias reduced estimators for synthetic data, with 2 monitor nodes chosen uniformly at random, $n$ target nodes, and shortest path sampling used to approximate traceroute. Plots based on 100 trials, where data points correspond to trial with median $\ell_{2}$ error, and dotted region shows pointwise bounds on $90 \%$ of trials. 


\subsection{Preferential Attachment Graph}

The preferential attachment (PA) graph was proposed for a model of the internet and the world wide web by Barabási and Albert in [2], and this has generated a large body of subsequent research, although the validity of the model as a representation of the router graph or the AS graph has been questioned (see, for example, [3]). The estimator $\widehat{\delta_{\leq k}}$ does not perform particularly well on the PA graphs that we used in our experiments, generating $\ell_{2}$ error that is sometimes smaller and sometimes larger than the biased estimator (see Table 2).

The most interesting detail of this series of experiments is the shape of the degree distribution estimated by $\widehat{\delta_{\leq k}}$. When plotted on a log-log scale (Figure $2 \mathrm{~b}$ ), the biased estimate of the degree distribution appears to be straight line, although with a different slope than the underlying distribution (this is consistent with the theoretical results of [1]). However, the "biased reduced" estimate appears to fall off faster than linear (when plotted on a log-log scale). This is typical of the experiments we conducted with other parameter settings for the PA graph. It could be an effect of the instance sizes being too small, but it persists over two orders of magnitude. Thus, it seems that locally non-tree-like aspects of the PA graph are decreasing the accuracy of $\overline{\delta_{\leq k}}$. As shown in Figure 1 and to be elaborated upon in Section 5 , the degree distribution of the AS graph does not fall off faster than linear when estimated with $\widehat{\delta_{\leq k}}$. This could mean that the shortest path routing used in the experiment is not a close enough approximation of the true traceroute sampled paths. But it could be interpreted as additional evidence that the AS graph is not distributed according to the PA graph process.

\begin{tabular}{|r|r|r|r|r|r|}
\hline$n$ & $m$ & $n_{m}$ & $n_{t}$ & $\%$ err $_{\text {biased }}$ & \% err \\
\hline 1,000 & 5 & 2 & $n / 8$ & 2.29 & 2.35 \\
& & & $n / 2$ & 1.95 & 2.66 \\
& & & $n$ & 1.71 & 2.88 \\
& & 8 & $n / 2$ & 1.26 & 2.00 \\
& & 16 & $n / 2$ & 0.91 & 1.57 \\
\hline 10,000 & 10 & 2 & $n / 8$ & 3.47 & 2.36 \\
& & $n / 2$ & 3.23 & 3.39 \\
& & & $n$ & 3.03 & 4.31 \\
\hline 100,000 & 15 & 2 & $n$ & 3.99 & 4.43 \\
\hline
\end{tabular}

Table 2. $\ell_{2}$ error in degree distribution estimation with and without bias reduction for Preferential Attachment graph with $n$ nodes and $m$ out-edges per node, $n_{m}$ monitors and $n_{t}$ targets (median values of 100 trials).

\subsection{Random Geometric Graph, $G(\mathcal{X} ; r)$}

For graphs with high clustering coefficient, the proof sketched in Section 3 will not apply. However, the traceroute paths found by skitter exhibit some level of clustering. To investigate the performance of the bias-reduction technique on graphs with clustering, we examine random geometric graphs $G(\mathcal{X} ; r)$. These graphs are formed by selecting a set of $n$ points independently and uniformly at random from the unit square, and linking two points with an edge if and only if they are within $\ell_{2}$ distance $r$ (for a detailed treatment, see [16]). The performance of the bias-reduction technique is summarized for a variety of geometric random graphs in Table 3 .

The plot exhibited in Figure 2c is typical for the performance of bias reduction on random geometric graphs; although the bias-reduced estimate is closer to the truth, it is still quite far away from it. The tail of the estimated ccdf, with or without bias reduction, falls off noticeably more slowly than that of the true degree distribution, and looks more like a power-law than it should. 
In light of this, it seems that future research should investigate the amount of clustering present in the AS graph. This will permit us to better gauge the accuracy of the bias-reduced estimate of the degree distribution there. However, understanding clustering in the AS graph is hard for the same reasons that understanding the degree distribution is hard, which is due to the lack of unbiased data.

\begin{tabular}{|r|r|r|r|r|r|}
\hline$n$ & $d$ & $n_{m}$ & $n_{t}$ & $\operatorname{err}_{\text {biased }}$ & err $_{\text {reduced }}$ \\
\hline 1,000 & 15 & 2 & $n / 8$ & 3.14 & 2.77 \\
& & & $n / 2$ & 2.91 & 2.49 \\
& & & $n$ & 2.73 & 2.17 \\
& & 8 & $n / 2$ & 2.45 & 2.50 \\
& & 16 & $n / 2$ & 2.23 & 2.49 \\
\hline 10,000 & 20 & 2 & $n / 8$ & 3.87 & 3.57 \\
& & & $n / 2$ & 3.68 & 3.36 \\
& & & $n$ & 3.55 & 3.16 \\
\hline 100,000 & 25 & 2 & $n$ & 4.19 & 3.90 \\
\hline
\end{tabular}

Table 3. $\ell_{2}$ error in degree distribution estimation with and without bias reduction for geometric random graph, $G(\mathcal{X}, r)$ where $d=\pi r^{2} n$, with $n_{m}$ monitors and $n_{t}$ targets (median values of 100 trials).

\subsection{Western States Power Graph}

In addition to studying the behavior of bias reduction on the random graphs describe above, we also consider the estimator's performance on synthetic data that is based on a network from the real world, the Western States Power Graph (WSP Graph) [22]. This graph represents the power transmission links between 4,941 nodes, representing the generators, transformers, and substations in the Western United States. It is roughly similar in size to the AS graph, and also similar because both networks represent real objects which are connected by real wires.

The result of the bias-reduction technique is shown in Figure $2 \mathrm{~d}$. The $\ell_{2}$ error is higher after bias reduction, but this is because the bias-reduction technique filters out all vertices of degree less than 3 . Since these low degree vertices are prevalent in the WSP graph, we also compare the bias-reduced estimate to the degree distribution of the WSP graph restricted to vertices of degree 3 and higher. Table 4 shows the unconditioned $\ell_{2}$ error for one experiment, and the $\ell_{2}$ error of the estimated cdfs conditioned on vertices having degree at least 3 for a range of experiments.

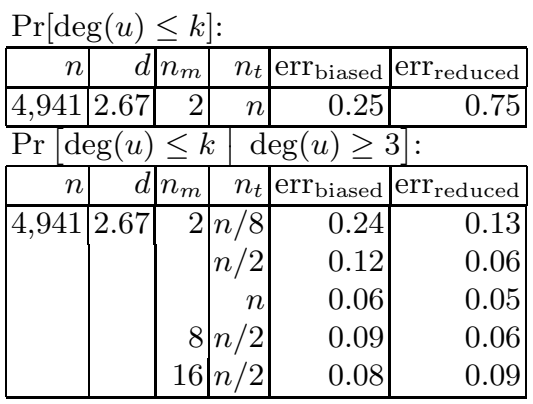

Table 4. $\ell_{2}$ error in degree distribution estimation with and without bias reduction for Western States Power Graph ( $n=4,941, m=6,594)$ with $n_{m}$ monitors and $n_{t}$ targets (median values of 100 trials). 


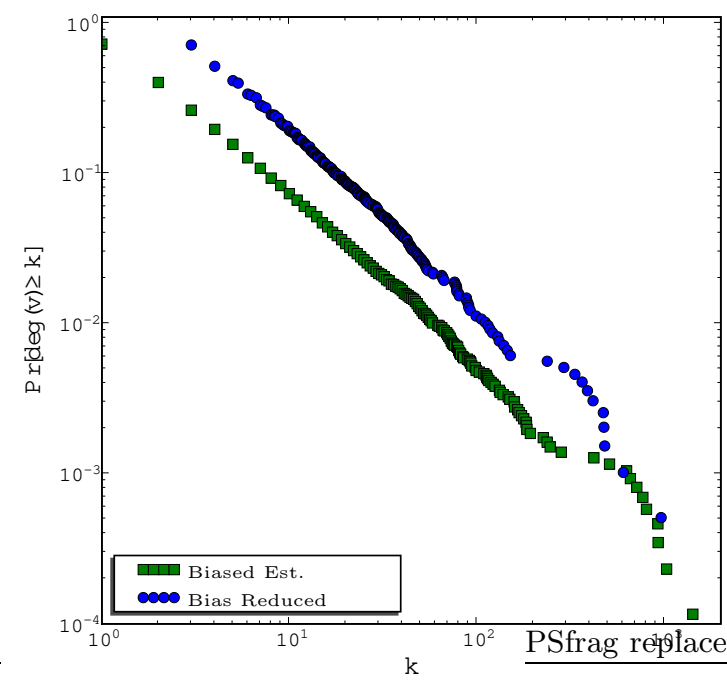

(a) CAIDA skitter, March, 2003.

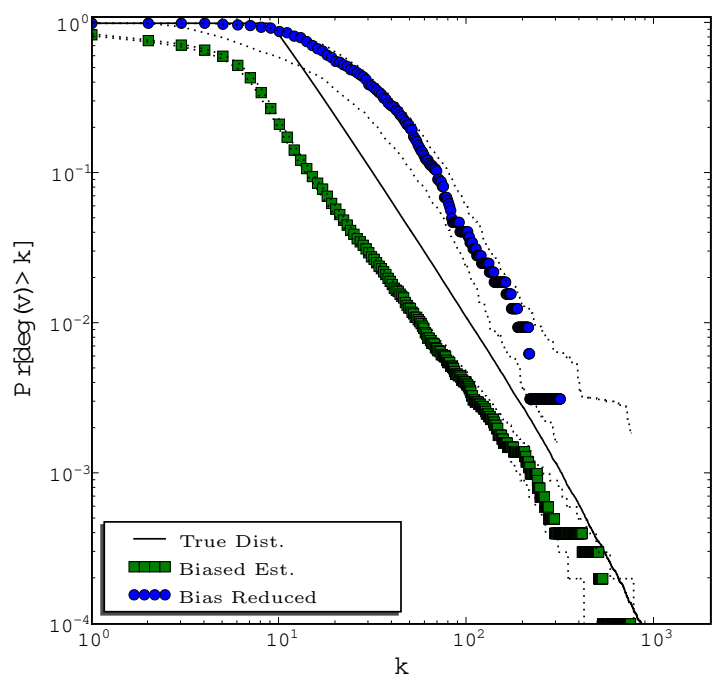

(b) PA graph.

Fig. 3. Estimated degree distribution ccdf of CAIDA skitter data from March, 2003 with and without bias reduction and estimated degree distribution ccdf of PA Graph with similar parameters $(n=10,000$ nodes, $m=10$ out-edges per node, 20 source nodes, $n / 2$ target nodes) with and without bias reduction. Both estimates of skitter data follow power law, but bias reduced estimate of PA Graph does not.

\section{$5 \quad$ AS Graph}

The previous two sections showed theoretically and by computer simulations that the bias-reduction technique developed in Section 2 can be an effective way to reduce the errors introduced by traceroute sampling. This section reports on the results of applying the bias-reduction technique to traceroute-sampled data from the CAIDA skitter project.

A recent paper by Mahadevan, Krioukov, Fomenkov, Dimitropoulos, claffy, and Vahdat provides a detailed analysis of CAIDA skitter data from March, 2004 [14]. We follow the methodology used there, and, in particular, we aggregate the routes observed over the course of a month (from daily graphs provided by CAIDA), and we remove all AS-sets, multi-origin ASes, and private ASes, and discard all indirect links.

The results of applying the bias-reduction technique to the March, 2004 skitter data are plotted in Figure 1. This data set contains 9,204 nodes and 28,959 edges, so the average degree before bias reduction is 6.29. There are 22 ASes in the monitor set, and between $10 \%$ and $50 \%$ of ASes are represented in the target set. The bias-reduction technique yields an estimate of $\widehat{\operatorname{deg}}(u)<\infty$ for 2, 078 vertices, and the average degree after bias reduction is 12.66 (which is very close to 12.52 , the biased average degree of vertices with degree at least 3).

The behavior of the bias reduced estimate for $k$ values around 64 is particularly interesting (see Figure 1). Although it is far from definitive, the lack of ASes with degree between 65 and 90 could be the result of economic or technological factors. For example, the Juniper T320 edge router has the ability to house up to 64 interfaces in one chassis. This, or similar product specifications, could lead AS operators to avoid connecting to slightly more than 64 other ASes.

Finally, the fact that the bias reduced estimate does not fall off at a superlinear rate provides some additional evidence against the theory that the AS graph is an example of a preferential attachment model (see comparison in Figure 3). This argument has been made previously based on completely different considerations (see, for example, [3]). 


\section{Conclusion}

In this paper we introduced a new approach to addressing the bias inherent to traceroute sampling. Starting from the multiple-recapture population estimation technique of statistics, we developed a bias reduction technique applicable to the highly dependent random variables present in path sampling.

In an idealized theoretical framework of shortest path sampling in Erdős-Rényi graphs, we described how to rigorously prove that the proposed estimator is asymptotically unbiased, and, using computer experiments, we show that the estimator can give significant improvements when the target nodes constitute a fraction of vertex set. Computer experiments also highlighted some of the weak points of this estimator, including the less-than-perfect estimates on locally non-tree-like graphs, like the PA graph and the random geometric graph.

Applying the bias-reduction technique to the CAIDA skitter data provided new evidence that the AS graph is not a preferential attachment graph, and also uncovered a way that economic and technological limitations are reflected in the AS degree distribution.

The theoretical and computer simulations supporting the effectiveness of the bias-reduction technique all rely on the assumption that shortest path routing is a close-enough approximation of BGP routing. This assumption should be considered in more detail, and the behavior of the bias-reduction technique under a more realistic model of traceroute is an important future direction of research.

\section{Acknowledgements}

ADF would like to thank Josh Grubman for pointing us towards the specifications of the Juniper T320 router, even if he does not believe that product specifications are likely to result in the absence of nodes with degree slightly above 64 .

\section{References}

1. D. Achlioptas, A. Clauset, D. Kempe, and C. Moore. On the bias of traceroute sampling: or, power-law degree distributions in regular graphs. In STOC '05: Proceedings of the thirty-seventh annual ACM symposium on Theory of computing, pages 694-703, New York, NY, USA, 2005. ACM Press.

2. A.-L. Barabási and R. Albert. Emergence of scaling in random networks. Science, 286(5439):509-512, 1999.

3. Q. Chen, H. Chang, R. Govindan, S. Jamin, S. J. Shenker, and W. Willinger. The origin of power laws in Internet topologies revisited. In INFOCOM 2002, Twenty-First Annual Joint Conference of the IEEE Computer and Communications Societies, Proceedings, volume 2, pages 608-617, 2002.

4. A. Clauset and C. Moore. Accuracy and scaling phenomena in internet mapping. Physical Review Letters, 94(1):018701, 2005.

5. L. Dall'Asta, I. Alvarez-Hamelin, A. Barrat, A. Vazquez, and A. Vespignani. A statistical approach to the traceroute-like exploration of networks: theory and simulations. LNCS, 3405:140, 2005.

6. P. Erdős and A. Rényi. On random graphs. I. Publ. Math. Debrecen, 6:290-297, 1959.

7. M. Faloutsos, P. Faloutsos, and C. Faloutsos. On power-law relationships of the internet topology. In SIGCOMM '99: Proceedings of the conference on Applications, technologies, architectures, and protocols for computer communication, pages 251-262, New York, NY, USA, 1999. ACM Press.

8. O. Frank. A survey of statistical methods for graph analysis. Sociological Methodology, 12:110-155, 1981.

9. J.-L. Guillaume, M. Latapy, and D. Magoni. Relevance of massively distributed explorations of the internet topology : Qualitative results. Computer networks, 50(16):3197-3224, 2006.

10. k c claffy, T. E. Monk, and D. McRobb. Internet tomography. Nature, January 1999.

11. A. S. Klovdahl. The Small World (in honor of Stanley Milgram), chapter Urban social networks: Some methodological problems and possibilities. ABLEX, 1989.

12. A. Lakhina, J. Byers, M. Crovella, and P. Xie. Sampling biases in ip topology measurements. In 22nd Annual Joint Conference of the IEEE Computer and Communications Societies (INFOCOM 2003), volume 1, pages 332-341. IEEE, April 2003.

13. J. Leguay, M. Latapy, T. Friedman, and K. Salamatian. Describing and simulating internet routes. In 4th International IFIP-TC6 Networking Conference, Waterloo, Canada, May 2-6, 2005. Proceedings, volume 3462 of Lecture Notes in Computer Science, pages 659-670, 2005. 
14. P. Mahadevan, D. Krioukov, M. Fomenkov, X. Dimitropoulos, k c claffy, and A. Vahdat. The internet AS-level topology: three data sources and one definitive metric. SIGCOMM Comput. Commun. Rev., 36(1):17-26, 2006.

15. J.-J. Pansiot and D. Grad. On routes and multicast trees in the internet. SIGCOMM Comput. Commun. Rev., 28(1):41-50, 1998.

16. M. Penrose. Random geometric graphs, volume 5 of Oxford Studies in Probability. Oxford University Press, Oxford, 2003.

17. T. Petermann and P. D. L. Rios. Exploration of scale-free networks. European Physical Journal B, 38:201-204, 2004.

18. I. Pickands, J. and M. Raghavachari. Exact and asymptotic inference for the size of a population. Biometrika, 74(2):355-363, 1987.

19. G. Pólya. Probabilities in proofreading. Amer. Math. Monthly, 83(1):42, 1975.

20. M. J. Salganik and D. D. Heckathorn. Sampling and estimation in hidden populations using respondent-drive sampling. Sociological Methodology, 34:193-239., 2004.

21. F. Viger, A. Barrat, L. Dall'Asta, C. Zhang, and E. Kolaczyk. Network Inference from TraceRoute Measurements: Internet Topology 'Species'. Arxiv preprint cs.NI/051000\%, 2005.

22. D. J. Watts and S. H. Strogatz. Collective dynamics of "small-world" networks. Nature, 292:440-442, 1998. 